\title{
Beyond vibrational self-consistent-field methods: Benchmark calculations for the fundamental vibrations of ethylene
}

\author{
Ove Christiansen* and Josep M. Luis ${ }^{\dagger}$ \\ Department of Chemistry, University of Arhus, DK-8000 Arhus C, Denmark.
}

\begin{abstract}
A number of different methods for calculation of vibrational energies are reviewed and tested for calculation of the fundamental vibrational frequencies for ethylene. The methods tested for the description of the vibrational states are vibrational self consistent field (VSCF), vibrational configuration interaction (VCI), vibrational Møller-Plesset theory (VMP), and vibrational coupled cluster (VCC). The convergence of the different methods towards the full vibrational configuration interaction (FVCI) result is discussed for a modest-sized one-mode basis. For larger one-mode basis sets results are presented for selected methods. Different representations of the potential energy surface are compared including full and partial quartic force field as well as expansions using up to sixth derivatives of the potential energy surface. Using MP2 electronic structure theory the electronic structure basis set convergence is tested.
\end{abstract}

\footnotetext{
* Electronic mail : ove@chem.au.dk

$\dagger$ Permanent address: Institute of Computational Chemistry and Department of Chemistry, University of Girona, Campus de Montilivi, 17071 Girona, Catalonia, Spain. Electronic mail: josepm.luis@udg.es
} 


\section{INTRODUCTION}

Molecular vibrations are directly or indirectly important in many disciplines of chemistry ${ }^{1}$ including high-resolution vibrational spectroscopy, UV spectroscopy, photo-electron spectroscopy etc. Vibrational energies are important for chemical reactions as sources and sinks. The interpretation of femto-second laser experiments also typically involves vibrational states - the only difference is now that the behavior of superposition states is central. ${ }^{2}$ Vibrational motion is important for molecular properties causing both an averaging of electronic properties over the vibrational motion as well as pure vibrational contributions to the properties at hand, as for example has been demonstrated for non-linear optical properties. ${ }^{3}$

Perturbation theory using harmonic oscillator reference states has long been a standard approach for theoretical vibrational spectroscopy. However, there are many problems where perturbation theory is seriously challenged, for example due to large amplitude motion and/or multiple minima. Methods under the general label of "Variational methods" have been developed over the years to be able to address more general problems. References to many different approaches can be found in the compilation in Ref.1. In this paper we shall consider methods that have the vibrational self-consistent field (VSCF) approach as their natural starting point. Methods based upon $\mathrm{VSCF}^{4-7}$ has gained increasing popularity in recent years for the calculation of anharmonic vibrational states. In the VSCF approach each degree of freedom vibrates in the average field of the other modes. The neglect of direct correlations has consequences on the final accuracy that can be achieved and a number of different methods have been suggested for improving the accuracy. Vibrational configuration interaction $(\mathrm{VCI})^{8-10}$ has been one of the approaches used for including the correlations between the different degrees of freedom. Another branch of active research in recent years has been vibrational Møller-Plesset ${ }^{11}$ perturbation theoretical methods, where the reference for the perturbation treatment is now the VSCF state, rather than simply the harmonic oscillator states. ${ }^{12-15}$ The ideas of VMP and VCI have also been developed by other groups in different context. ${ }^{16-18}$ In a recent publication ${ }^{19}$ a second quantization (SQ) formulation of many-mode dynamics was outlined. Based on this formalism a new vibrational coupled cluster (VCC) method was devised ${ }^{19,20}$ and tested with encouraging results.

In this paper we shall discuss and compare the performance of VSCF, VMP, VCI and VCC methods for the prediction of the fundamental vibrations in ethylene. Ethylene is a 
rather well studied system and for the electronic ground state many issues concerning the fundamental vibrations are well known and have been studied in a number of theoretical works. ${ }^{16,21-25}$ Nevertheless, we choose to consider this system in our calculations since 1). It is small enough to allow us to perform true benchmark studies and learn about the convergence of various effects. 2). It is the smallest system among a number of related organic molecules which we shall study in forthcoming papers. Among the problems studied in this paper are the importance of vibrational correlation and the size and nature of the normal coordinate one-mode basis for the vibrational calculations. The representation of the potential energy surface with respect to expansion to different orders and inclusion/exclusion of many-mode couplings will also be considered as well as the importance of the numerical errors in the calculation of the numerical derivatives. The convergence of the atomic basis set used in the electronic structure calculations behind the PES will be discussed briefly, though benchmarking of the electronic structure part is not a primary target of this paper since a number of aspects have been discussed in previous studies. ${ }^{21,22}$

In Sec. II the general aspects of the theories are briefly reviewed and compared. Section III describes more technical aspects of the computations. The results from the calculations on $\mathrm{C}_{2} \mathrm{H}_{4}$ are discussed in Sec. IV. Finally, in section $\mathrm{V}$ we conclude with a summary.

\section{THEORY}

\section{A. Vibrational self consistent field theory}

Consider a system with $M$ modes, where a "mode" denotes a vibrational degree of freedom. In this paper the modes are standard normal coordinates. In the vibrational self consistent field (VSCF) model one seeks to find the optimal one-mode functions when the $M$-mode wave function is a simple product of one-mode functions.

$$
\Phi_{\mathbf{i}}\left(q_{1}, q_{2}, \ldots, q_{M}\right)=\prod_{m=1}^{M} \phi_{i^{m}}^{m}\left(q_{m}\right)
$$

Thus, VSCF is essentially just the application of the Hartree approximation to the problem of coupled distinguishable anharmonic oscillators. The one-mode functions for each mode, $\phi_{i^{m}}^{m}\left(q_{m}\right)$, with the normalized one-mode condition $\left\langle\phi_{i^{m}}^{m}\left(q_{m}\right) \mid \phi_{j^{m}}^{m}\left(q_{m}\right)\right\rangle=\delta_{i_{m} j_{m}}$ are found by 
applying variational criteria to the energy

$$
E_{\mathbf{i}}=\left\langle\Phi_{\mathbf{i}}|H| \Phi_{\mathbf{i}}\right\rangle
$$

The wave function and the energy are indexed by a vector, $\mathbf{i}$, indicating the nature of the VSCF reference state.

Working out the variational criteria in detail one obtains that the VSCF one-mode functions can be found as eigenfunctions from the effective one-mode eigenvalue equations

$$
F^{m, \mathbf{i}} \phi_{i^{m}}^{m}\left(q_{m}\right)=\epsilon_{i^{m}}^{m} \phi_{i^{m}}^{m}\left(q_{m}\right)
$$

where the following effective operator $F^{m, \mathbf{i}}$ has been defined for each mode

$$
F^{m, \mathbf{i}}=\left\langle\prod_{m^{\prime}=1, \neq m}^{M} \phi_{i^{m^{\prime}}}^{m^{\prime}}\left(q_{m^{\prime}}\right)|H| \prod_{m^{\prime \prime}=1, \neq m}^{M} \phi_{i^{m^{\prime \prime}}}^{m^{\prime \prime}}\left(q_{m^{\prime \prime}}\right)\right\rangle
$$

This is a mean-field operator for each mode $m$ showing clearly that the interaction between the modes are only included in a average sense. The total VSCF energy is obtained from Eq.(2) using the optimal $\phi_{i^{m}}^{m}\left(q_{m}\right)$. Using that the modals satisfy the VSCF eigenvalue equations, the one-mode eigenvalues of the effective operator can be written in terms of the Hamiltonian and the eigenfunctions as

$$
\epsilon_{i^{m}}^{m}=\left\langle\phi_{i^{m}}^{m}\left(q_{m}\right)\left|F^{m, \mathbf{i}}\right| \phi_{i^{m}}^{m}\left(q_{m}\right)\right\rangle=\left\langle\Phi_{\mathbf{i}}|H| \Phi_{\mathbf{i}}\right\rangle
$$

for all $i^{m}$ in $\mathbf{i}$. Since some terms may be constant for a given mode they may for efficiency be left out of that particular $F^{m, \mathbf{i}}$ and thus out of Eq.(3) therebye invalidating the last equality of Eq.(5). This does not change the general principles of the VSCF algorithm and the final VSCF energy calculated from Eq.(2).

The $\phi_{s^{m}}^{m}\left(q_{m}\right)$ functions are in this paper obtained from an expansion in terms of a set of Harmonic oscillator functions using normal coordinates. Other types of basis sets are possible as well as there are other approaches to the solution of the VSCF equations. The one-mode functions are denoted modals in analogy to the orbitals of electronic structure theory. The VSCF modals can be used in the calculation with more accurate wave function that includes the vibrational correlation. It seems reasonable to assume that for a given accuracy the number of basis functions required using VSCF modals is smaller than if the original harmonic oscillator basis are used. In this paper we shall use a rather large primitive 
harmonic oscillator basis which is computationally inexpensive in VSCF, and then use a limited number of VSCF modals constructed from this basis in the correlated calculations. For simplicity we use in this paper the same number, $N_{m}$, of VSCF modals per mode for all modes.

\section{B. Vibrational structure theory in second quantization}

A second quantization formulation for vibrational structure theory was developed in Ref.19. In this formulation both the relevant quantum mechanical states and the operators can be expressed in terms of creation and annihilation operators. The creation and annihilation operators for a mode $m$ relates to an orthonormal one-mode basis

$$
\left\{\phi_{s^{m}}^{m}\left(q_{m}\right), s^{m}=1, N_{m}\right\}
$$

The creation operator $a_{s^{m}}^{m, \dagger}$ creates the mode in a vibrational level with index $s^{m}$ for the mode $m$. The corresponding annihilation operator $a_{s^{m}}^{m}$ removes this vibrational level again. The creation and annihilation operators satisfy certain commutator relations, $\left[a_{r^{m}}^{m}, a_{s^{m^{\prime}}}^{m^{\prime} \dagger}\right]=\delta_{m m^{\prime}} \delta_{r^{m} s^{m}},\left[a_{r^{m}}^{m}, a_{s^{m^{\prime}}}^{m^{\prime}}\right]=0,\left[a_{r^{m}}^{m \dagger}, a_{s^{m^{\prime}}}^{m^{\prime} \dagger}\right]=0$, which defines the algebra in the second-quantization representation. Any Hartree-Product M-mode basis state can be represented in terms of the creation operators as

$$
|\mathbf{r}\rangle=\prod_{m=1}^{M} a_{r^{m}}^{m \dagger}|\mathrm{vac}\rangle
$$

where $|v a c\rangle$ is the state vector where all modes are unoccupied in all levels.

The Hamiltonian operator can also be written in second quantization algebra. We shall in this paper use a sum over product form for the Hamiltonian which in both first and second quantization can be written as

$$
H=\sum_{t=1}^{N_{t}} c_{t} \prod_{m=1}^{M} h^{m, t}
$$

This form for the Hamiltonian has the usual truncated expanded force field representation of the potential as a special case where for the potential the one-mode operators in the factors are all of the form $q_{m}^{i}$, with $\mathrm{i}=1,2,3,4$ (as well as the unit operator for the remaining modes). Both diagonal and non diagonal terms of a quartic multimode potential are thus included. In addition there are terms corresponding to the kinetic energy operator. The 
difference between first and second quantization lies in the representation of the one-mode operators. The one-mode operators in SQ notation are

$$
{ }^{S Q} h^{m, t}=\sum_{r^{m}, s^{m}} h_{r^{m} s^{m}}^{m, t} a_{r^{m}}^{m \dagger} a_{s^{m}}^{m}
$$

which is related to the first quantization representation through the matrix elements

$$
h_{r^{m} s^{m}}^{m, t}=\left\langle\phi_{r^{m}}^{m}\left(q_{m}\right)\left|h^{m, t}\right| \phi_{s^{m}}^{m}\left(q_{m}\right)\right\rangle
$$

where $h^{m, t}$ are the first quantization one-mode operators.

The SQ formulation allows a convenient formulation for coupled cluster wave vibrational wave functions. ${ }^{20}$ In the subsequent subsections different correlation methods are described.

\section{Vibrational configuration interaction wave functions}

Explicit interactions between the modes are ignored in VSCF. Including such interactions requires a wave function beyond a simple Hartree Product. Thus excitations from occupied modals into "virtual" modals - the unoccupied levels in the VSCF - must be included. These excitations can be generated by excitation operators denoted $\tau_{\mu}$. Accordingly the full configuration space may be written as $\left\{\left|\Phi_{\mathbf{i}}\right\rangle, \tau_{\mu}\left|\Phi_{\mathbf{i}}\right\rangle\right\}$. The $\mu$ index is a compound index giving all necessary information to specify the excitation including which modes are excited and to which levels. The excitations can be written as products of one mode excitations of the type, $a_{a^{m}}^{m \dagger} a_{i^{m}}^{m}$ for excitation from the occupied level $i^{m}$ to the unoccupied level, $a^{m}$. The

vibrational configuration interaction $(\mathrm{VCI})^{5,6,8-10}$ parameterization of the wave-function is a linear expansion in the physical space of all Hartree-Products for the $M$-mode system

$$
|\mathrm{VCI}\rangle=\mathrm{C}_{\mathbf{i}}\left|\Phi_{\mathbf{i}}\right\rangle+\sum_{\mu} \mathrm{C}_{\mu} \tau_{\mu}\left|\Phi_{\mathbf{i}}\right\rangle
$$

The parameters are determined from variational criteria giving the usual CI eigenvalue equations. Including the full sum over all excitations $\mu$ the Full VCI (FVCI) wave function is obtained. The FVCI wave function is the exact wave function for the given Hamiltonian and one-mode basis set. The difference between the VSCF energy and the FVCI energy is denoted the correlation energy.

For obvious reasons an unavoidable approximation for larger systems is using a limited number of Hartree-Products (a restricted M-mode basis) of the full set that could be constructed from a given one-mode basis. In this paper we have checked the convergence of 
our results with respect to such truncations. Truncated VCI treatments include only a part of the full excitation space and give accordingly only a fraction of the correlation energy but can be computationally much cheaper. One way to introduce approximations to the FVCI wave function is to truncate the VCI expansion at some excitation level lower than the number of modes. Other ways of restricting the VCI space is possible, but the above approach is chosen for simplicity in relation to the following discussions and for efficiency in the calculations.

Two variants of VCI truncated by excitation level shall be considered here. In the first simpler case one use the same set of modals for all states. For example in the calculations denoted VCI [gs,n] we use the ground state VSCF modals in a VCI calculation with up to n-modal excitations, where the excitation are counted with respect to the ground state. A hierarchy of VCI models, VCI[gs,1], VCI[gs,2], VCI[gs,3], VCI[gs,4], .. , FVCI is obtained in this way. The other variant of VCI is denoted state-specific. In state-specific VCI each state has its own VSCF reference state, and for VCI[ss,n] wave functions the excitations are counted with respect to that reference state. Since the different VCI[ss] states have different modal basis sets they are not orthogonal.

Both the ground state and state-specific VCI have been discussed in a number of papers. ${ }^{5,6,8-10,20}$ In our implementation both types of VCI calculations are carried out using an iterative direct VCI methodology in conjunction with a Davidson algorithm as described in a previous paper $^{20}$.

\section{Vibrational Møller-Plesset perturbation theory}

Vibrational Møller-Plesset theory is the application of Møller-Plesset perturbation theory $^{11}$ to vibrational problems using a VSCF reference state. ${ }^{12-15}$ Thus, the zeroth order Hamiltonian $H_{0}$ and the corresponding zeroth order wave function are obtained from the VSCF mean-field description

$$
\begin{gathered}
H=H_{0}+U=F^{\mathbf{i}}+U^{\mathbf{i}} \\
F^{\mathbf{i}}=\sum_{m} F^{m, \mathbf{i}}
\end{gathered}
$$

The $U^{\mathbf{i}}$ operator describes the difference between the true many-mode interaction and the VSCF mean field representation. This separation is unique to each state motivating the 
$\mathbf{i}$ index vector. For brevity the $\mathbf{i}$ index vector is left out in the following. An ordinary perturbation expansion in orders of $\mathrm{U}$ with the VSCF state as the zeroth order state gives a sequence of perturbational corrections to the VSCF energy for each state: VSCF, VMP2, VMP3, VMP4, .. etc. Here, the VSCF energy is in fact the VMP1 energy.

The VMP expansion is formally written as

$$
\begin{gathered}
E=\sum_{n=0}^{\infty} E^{(n)} \\
|\Psi\rangle=\sum_{n=0}^{\infty}\left|\Psi^{(n)}\right\rangle
\end{gathered}
$$

Introducing the perturbation expansion into the time-independent Schrödinger equation and collecting terms according to order $n$ we obtain

$$
\left(H_{0}-E^{(0)}\right)\left|\Psi^{(n)}\right\rangle=-U\left|\Psi^{(n-1)}\right\rangle+\sum_{k=1}^{n} E^{(k)}\left|\Psi^{(n-k)}\right\rangle
$$

From this equation, we can arrive at equations for the perturbed wave function and energies by projection on the reference state or its orthogonal complement. In summary, the perturbed energy can be obtained as

$$
E^{(2 n+i)}=\left\langle\Psi^{(n+i-1)}|U| \Psi^{(n)}\right\rangle-\sum_{k=1}^{2 n+i-2} \sum_{m=\max (1, n+i-k)}^{\min (n+i-1,2 n+i-k-1)} E^{(k)}\left\langle\Psi^{(m)} \mid \Psi^{(2 n+i-m-k)}\right\rangle
$$

Here $i=0$ for even orders and $i=1$ for odd orders. The perturbed wave functions are obtained from

$$
\left|\Psi^{(n)}\right\rangle=P\left(H_{0}-E^{(0)}\right)^{(-1)} P\left[-U\left|\Psi^{(n-1)}\right\rangle+\sum_{k=1}^{n-1} E^{(k)}\left|\Psi^{(n-k)}\right\rangle\right]
$$

Here $\mathrm{P}$ is a projector to the space orthogonal to $\left|\Psi^{(0)}\right\rangle, P=1-\left|\Psi^{(0)}\right\rangle\left\langle\Psi^{(0)}\right|$. These equations can be implemented in a fairly general way in a VCI program. The VMP wave function and energies are thus calculated on the basis of the ability to calculate transformed vectors of the type

$$
\left|\rho^{(k)}\right\rangle=U\left|\Psi^{(k-1)}\right\rangle
$$

See Ref.15 for more on this particular approach to VMP and Refs.26-28 for similar approaches in electronic structure theory. VMP approaches was first pursued by Gerber and collaborators $^{12-14}$ in the second order variant under the name of correlation-corrected VSCF. 
Recently an open ended VMP approach was implemented based on direct VCI techniques allowing high order VMP calculations. ${ }^{15}$

As one proceeds to higher order in VMP the wave function includes more and more mode couplings in accord with the coupling of the modes in the Hamiltonian. Restricting the allowed number of modes coupled in the perturbed wave functions corresponds to restricting the vectors in Eq.(19) in a similar manner as in truncated VCI.

\section{E. Vibrational coupled cluster theory}

The vibrational coupled cluster ansatz is defined as

$$
|\mathrm{VCC}\rangle=\exp (T)\left|\Phi_{\mathbf{i}}\right\rangle
$$

Here $\left|\Phi_{\mathbf{i}}\right\rangle$ is a reference wave function, which in this paper is the VSCF state, and T is the so-called cluster operator. The cluster operator may be written as

$$
T=\sum_{\mu} t_{\mu} \tau_{\mu}
$$

The $t_{\mu}$ parameters are denoted the cluster amplitudes while $\tau_{\mu}$ are the corresponding excitation operators indexed by $\mu$. The excitation operators $\tau_{\mu}$ satisfy $\left[\tau_{\mu}, \tau_{\nu}\right]=0, \tau_{\nu}^{\dagger}\left|\Phi_{\mathbf{i}}\right\rangle=0$,

and $\langle\mu \mid \nu\rangle=\left\langle\Phi_{\mathbf{i}}\left|\tau_{\mu}^{\dagger} \tau_{\nu}\right| \Phi_{\mathbf{i}}\right\rangle=\delta_{\mu, \nu}$ where the manifold of excitations out of the $\left|\Phi_{\mathbf{i}}\right\rangle$ reference can be written as $|\mu\rangle=\tau_{\mu}\left|\Phi_{\mathbf{i}}\right\rangle$.

The VCC wave function ansatz is introduced into the time-independent Schrödinger equation and transformed with $\exp (-T)$.

$$
\exp (-T) H \exp (T)\left|\Phi_{\mathbf{i}}\right\rangle=E_{V C C}\left|\Phi_{\mathbf{i}}\right\rangle
$$

Projection onto the manifold of excitations out of the reference state $\langle\mu|=\left\langle\Phi_{\mathbf{i}}\right| \tau_{\mu}^{\dagger}$ gives a set of nonlinear equations for the cluster amplitudes

$$
0=e_{\mu}=\left\langle\mu|\exp (-T) H \exp (T)| \Phi_{\mathbf{i}}\right\rangle
$$

Projection onto the VSCF reference state gives the VCC energy as

$$
E_{V C C}=\left\langle\Phi_{\mathbf{i}}|\exp (-T) H \exp (T)| \Phi_{\mathbf{i}}\right\rangle
$$

which can be evaluated once the non-linear cluster equations have been solved for the cluster amplitudes. 
Limiting the space of allowed excitations corresponds to introducing approximate VCC methods. Thus, the sum over excitation in the definition of the cluster operator is truncated, as well as the cluster amplitude equations are only solved in the same excitation space. The VCC calculations reported in this paper are all state-specific. Thus, each calculation begins with the calculation of a VSCF state for each state, and thereafter an excitation space is defined based on excitations out of the VSCF reference state.

The advantage of VCC and its associated exponential parameterization is that it ensures size-extensivity (see Ref.19,20 and references therein for discussions on size-extensivity). This means that VCC has a correct scaling of the energies, wave-function and properties with respect to the size of the system. The energy of two non-interacting systems will for example be the sum of the energies of the two systems. This is trivially fulfilled in exact theory. While it is also satisfied for the non-variational VMP and VCC methods, it is not satisfied by truncated VCI methods. In addition to the long experience from the electronic structure calculations on the importance of this issue, the VCC calculations that have been carried out ${ }^{19,20}$ show that the accuracy for the truncated VCC results is better than the one for their VCI counterparts with the same excitation space.

For a complete excitation space both VCI and VCC have the flexibilty to represent the exact state (for the given set of modals and Hamiltonian). This is an advantage over VMP, since convergence of the VMP perturbation series can not be guaranteed, and in fact there is little reason to believe that VMP should converge. ${ }^{15}$ Thus, even though lowest order VMP may be a cost efficient approach in some aspects, there will in general be limitations to the accuracy that can be obtained in VMP calculations.

At a certain excitation level a $\operatorname{VCC}[\mathrm{n}]$ and $\mathrm{VCI}[\mathrm{n}]$ has the same number of free parameters. Ideally VCI $[\mathrm{n}]$ and $\mathrm{VCC}[\mathrm{n}]$ should have formal operation counts of the same order of magnitude, but VCC[n] is significantly more complicated to implement than VCI[n]. Thus the existing implementation is not fully optimal yet, but does allow an iterative solution of the VCC equations for systems with many degrees of freedom. The initial implementation is described in Ref. 20. 


\section{POTENTIAL ENERGY SURFACES AND COMPUTATIONAL ASPECTS}

The potential energy surfaces were expanded in normal coordinates. Harmonic force constants were obtained analytically employing the Gaussian $03^{29}$ package at the MP2 level using Dunnings correlation consistent basis sets. ${ }^{30}$ Numerical differentiation of the analytical harmonic force constants with respect to normal coordinates was used to calculate the anharmonic force constants. A number of different PES variants were tested. $2 M$ Hessian calculations were required for the semi-quartic force-field, where $M$ is the number of normal modes. A semi-quartic force field denotes a force field with up to quartic terms, but excluding those quartic terms that have four different modes (of type ijkl). For the full quartic forcefield $M(M+1)$ Hessian calculations were required. For semi-quartic force-field the addition of Romberg corrections ${ }^{31}$ required additional $2 M$ Hessian calculations. The same $2 M$ extra Hessian calculations allow for the evaluation of 5 and 6 order terms with at most 3-mode couplings.

The VSCF reference is represented through a one-mode basis of harmonic oscillator eigenfunctions using normal coordinates. In all calculations we used harmonic oscillator basis functions including all functions up to harmonic oscillator level $n=20$ using the harmonic oscillator basis that corresponds to the quadratic terms of the potential at hand. We have checked that the change in the energy of the VSCF calculations is absolutely negligible when the number of harmonic oscillator basis functions is increased.

Following the VSCF calculation the number of modals can be limited to include only a number of the VSCF modals available per mode with the given basis. After defining the reference occupation and the modal space the allowed excitation space is defined by giving a maximum excitation level for both VCI, VCC and VMP (optionally). For VMP in addition a perturbation order defines the different levels of approximations.

The MidasCpp (Molecular Interactions, dynamics and simulation in $\mathrm{C}++/$ Chemistry program package) program is used for the vibrational structure calculations. ${ }^{32}$ 


\section{RESULTS}

\section{A. Benchmark calculations against FVCI in modest sized basis}

\section{The convergence of $V C I[g s, n]$}

In Table I we report FVCI fundamental vibration energies for ethylene using the MP2/ccpVTZ semi-quartic PES and a modal basis with 3 ground state VSCF modals per mode. This is a small, but reasonable basis as we shall see in the next subsection. The FVCI space consists of 531441 Hartree Products.

Included in Table I are also the deviation from the FVCI fundamental vibrational energies as obtained in a number of truncated VCI $[\mathrm{gs}, \mathrm{n}]$ and $\mathrm{VCI}[\mathrm{ss}, \mathrm{n}]$ methods. Focusing first on the $\mathrm{VCI}[\mathrm{gs}, \mathrm{n}]$ results it is seen that both the maximum absolute errors and the mean of the absolute errors converge to the FVCI results when the excitation level $\mathrm{n}$ is increased towards the maximum 12 for the FVCI. However, there are a few surprising features. It is seen that the deviations found for $n=1,2,3$ are roughly similar and even roughly similar to those of (state-explicit) VSCF. The Hamiltonian contains direct 3-mode couplings and at least 3-mode couplings are required to correlate the ground state wave function correct through first order. For the fundamental vibrations in the case of $\mathrm{VCI}[\mathrm{gs}, \mathrm{n}]$ one excitation level is used to achieve the excitation to the fundamental vibrational level. Accordingly, only at the VCI $[\mathrm{gs}, 4]$ level convincingly more accurate results obtained is obtained. Errors of close to $10 \mathrm{~cm}^{-1}$ persist among the $\mathrm{CH}$-strech fundamentals even up to the VCI[gs,5] level. At the FVCI level the excited states related with CH-strech fundamentals have significant contributions from Hartree-Products that have two or more modes excited. Thus, to describe the CH-strech fundamental vibrations accurately also these mixing states must be describe accurately, and this slows down and causes sometimes some oscillations in the convergence towards FVCI. In such cases the wave function obtained for truncated VCI can be very different from the FVCI wave function. For instance, for the state with FVCI energy at $3141.80 \mathrm{~cm}^{-1}$ the corresponding $\mathrm{VCI}[\mathrm{gs}, 2]$ wave function is completely dominated by a single Hartree-Product, while with higher excitation levels there is substantial mixings between the fundamental vibration and a two-mode excitation. Thus, at this energy there are two closelying states that are completely mixed. At the $\mathrm{VCI}[\mathrm{gs}, 6]$ and $\mathrm{VCI}[\mathrm{gs}, 7]$ levels the maximum errors relative to FVCI[gs] for all states are less than $0.3 \mathrm{~cm}^{-1}$ and $0.02 \mathrm{~cm}^{-1}$ 
respectively.

\section{The convergence of VCI $[s s, n]$}

Consider now the state-specific VCI results included in Table I. First, it should be noted that the state specific FVCI[ss] results are different from the ground state FVCI[gs] results. This difference is solely due to that different VSCF modals are used in the state-specific calculations. The 21 one-mode basis functions per mode is transformed to VSCF modals for all modes and only 3 of these are used in the FVCI benchmark calculations. In FVCI[gs] the 3 modals are the 3 lowest ground state VSCF modals, while in FVCI[ss] the 3 modals per mode in the excited state calculations are those obtained from the corresponding VSCF excited state calculation. Comparing the FVCI[gs] and FVCI[ss] results we see thus the effect of using modals optimized for the excited state, when a modest sized basis is used. Using only 3 modals per mode the differences are typically $1-3 \mathrm{~cm}^{-1}$, but for a single $\mathrm{CH}$ stretch frequency it is of order $10 \mathrm{~cm}^{-1}$. As the modal basis of the FVCI calculations is increased towards the size of the primitive basis this difference of course vanish.

As for the ground state VCI calculations we see convergence in the VCI[ss,n] results with increasing n. Again, it is necessary to go to the $n=4$ level to obtain major reductions in the errors. At the level of at most 2-mode excitations the mean error of $\mathrm{VCI}[\mathrm{ss}, 2]$ is seen to be smaller than that of $\mathrm{VCI}[\mathrm{gs}, 2]$ but the maximum errors are actually increased. The state specific $\mathrm{VCI}[\mathrm{ss}, 3]$ results are rather accurate for 8 out of the 12 fundamentals having a mean $(\max )$ error of $1.40(2.81) \mathrm{cm}^{-1}$ which is a major improvement compared to $\mathrm{VCI}[\mathrm{gs}, 3]$. The 4 troublesome modes are the $\mathrm{CH}$-stretch vibrations. Also for the state specific calculations it is necessary to include up to 6-mode couplings to obtain results within $1 \mathrm{~cm}^{-1}$ of the FVCI results for all modes.

Inspecting the FVCI[ss] solution vectors a difference between the $\mathrm{CH}$-strech modes and the 8 lower lying modes is seen. While the 8 lower lying modes have FVCI wave functions with more than $90 \%$ overlap (the overlap $=\langle V S C F \mid F V C I\rangle$ ) with the VSCF reference state, the $4 \mathrm{CH}$-stretch modes have significantly smaller overlaps (down to about $60 \%$ ). Thus, these states have large contributions from one or more Hartree-Products different from the VSCF reference state. Clearly, using one Hartree-Product to optimize the modals and define the excitation space is less optimal for these states compared to the lower more pure single- 
reference states. This does not hinder convergence in the results of the VCI calculations when the excitation space is increased, but slows it down. In fact it also in some cases causes the numerical optimization of the VCI states to be slower.

\section{The VMP results}

In Figure 1 are given the logarithms of the contributions to the total energies for the ground and fundamental vibrations for VMP methods in different orders. It is seen that even though many of these states have convergent VMP series others have divergent VMP series already for this rather modest basis set. The 4 obviously divergent states in Figure 1 are the $4 \mathrm{CH}$-stretch fundamentals. Divergencies for VMP were also found in a previous study. ${ }^{15}$ In that work it was also found that the convergence properties of the perturbation series were critically dependent on the choice of basis. Thus, it is very likely that increasing the basis set size will make the convergence problems even worse effecting potentially also more states. However, Figure 1 shows already clearly that one can not use VMP as a vehicle for obtaining arbitrarily high accuracy in the prediction of vibrational energies.

Having discussed some of the problems in using higher order VMP methods also for ethylene it should be recalled that divergences in high order perturbation theory does not necessarily hinder low order VMP results from being very useful from a pragmatic point of view. Some selected low order VMP results are included in Table II. In addition to the full VMP2 and VMP4 results denoted as VMP2[3] and VMP4[6] we give also some restricted VMP results. For example VMP2[2] where the perturbed wave function is only allowed to have single and double mode-couplings. We see, that the full VMP2 approach (=VMP2[3]) does remarkably well compared to the VCI[gs,3] and VCI[ss,3] calculations with a similar number of parameters, with smaller errors in both mean and maximum sense. The improvements are primarily due to the $\mathrm{CH}$-stretch modes, since for the other modes $\mathrm{VCI}[\mathrm{ss}, 3]$ is more accurate than VMP2. Imposing limitations in the allowed excitation leads clearly to less accurate result compared to the full VMP models. The full VMP4 approach (including up to 6-mode couplings) has reasonably high accuracy for many states. However, a similar accuracy was obtained in the VCI calculations with much fewer parameters and some states are observed to behave somewhat problematic for VMP4. 


\section{VCC results}

The state-specific VCC results are included in Table II. The VCC[ss,1] results are equivalent to the $\mathrm{VSCF}=\mathrm{VCI}[\mathrm{ss}, 1]$ results. At the level of at most double excitations the deviations of VCC[ss,2] compared to FVCI[ss] are seen to be rather similar to those of VCI[ss,2]. Proceeding from $\mathrm{VCC}[\mathrm{ss}, 2]$ to $\mathrm{VCC}[\mathrm{ss}, 3]$ we make the same observation as in the state specific VCI calculations. For all fundamentals except the CH-stretch modes the errors are significantly reduced. Indeed for these states the accuracy obtained by $\mathrm{VCC}[\mathrm{ss}, 3]$ is better than any other method with similar or less parameters. The mean and max errors of these 8 fundamental vibrations are about 1 and $1.5 \mathrm{~cm}^{-1}$ respectively.

The errors in the CH-stretch fundamentals are much larger though they are still smaller than the VCI errors for the same number of free parameters. The practical solution of the non-linear VCC equations were actually quite problematic for these states. The larger errors relative to the other states and the problems in converging the equations for these states are assumed to have a common origin, namely the fact that these states have large contributions from one or more Hartree-Products different from the VSCF reference state. This problem was already found in the state specific VCI calculations. In the case of VCC it makes the non-linear VCC equations sometimes very difficult to solve using the iterative solution algorithm implemented so far. We are currently improving the VCC algorithm to handle these issues, before embarking on the larger excitation space VCC calculations.

\section{B. Convergence of the one-mode basis}

In Table III is given the results obtained for different restrictions on the one-mode basis. In all cases the restrictions are based on limiting the number of modals in the VCI[gs,4] to be only $N_{m}$ number of modals per mode. It is seen that that fundamental vibrational energies are relatively fast convergent, and it seems that the $N_{m}=9$ results are good enough as reference with the given number of digits. Thus, the previous FVCI results with $N_{m}=3$

probably entails basis set errors of order $1-27 \mathrm{~cm}^{-1}$. At $N_{m}=5$ the mean (max) errors are down to $0.36(1.20) \mathrm{cm}^{-1}$ compared to the $N_{m}=9$ results. 


\section{The representation of the potential energy}

The representation of the potential energy surface were analyzed in a number of different ways. Some studies of the convergence of the PES with respect to how the derivatives of the PES were calculated and how many terms is included in the expansion are given in Table IV. In this Table fundamental vibration are given for various representation of the PES studied using VCI[ss,4] and a $N_{m}=9$ modal basis. The representations of the PES include semiquartic with only up to 3-mode couplings, full quartic including up to 4-mode couplings, and semi-quartic with additional fifth and sixth order terms in the polynomial expansion. Furthermore, the level of numerical noise was tested by using Romberg interpolation in the calculation of the semi-quartic force field. It was found that the level of noise was rather small - about one tenth of an $\mathrm{cm}^{-1}$.

The differences between the semi-quartic and full quartic forcefield were modest, less than $3 \mathrm{~cm}^{-1}$, for fundamental vibrations, apart from the four $\mathrm{CH}$-stretch modes were the 4mode coupling terms increased the fundamental vibrations by $11-26 \mathrm{~cm}^{-1}$. Including higher

order terms in the 3-mode coupling part gave a reduction of the $\mathrm{CH}$-stretch frequencies of similar magnitude. The other fundamentals were affected by up to $10 \mathrm{~cm}^{-1}$. Of course the magnitude of these effects is very system specific and the importance of the higher terms can be dramatically different for a more floppy molecule.

\section{Dependence on electronic structure calculation}

We have inspected the dependence on the basis set used in the electronic structure calculations at the MP2 level. Higher level electron correlation treatments are certainly possible, but in the literature a number of other calculations including $\operatorname{CSD}(\mathrm{T})$ calculations ${ }^{21,22}$ already exist and we have focused on other issues in this paper.

The harmonic frequencies obtained in MP2 calculations with various basis sets and in the $\operatorname{CCSD}(\mathrm{T})$ calculations of Refs. 21,22 are given in Table V. The fundamental frequencies obtained from some of the different MP2 PES are given in Table VI. The vibrational calculations were carried out at the VCI[gs,4] level using $N_{m}=9 \mathrm{VSCF}$ modals per mode.

Proceeding from MP2/cc-pVTZ to MP2/cc-pVQZ the harmonic frequencies changes less than $5 \mathrm{~cm}^{-1}$. The change in the fundamentals is generally of a similar order of magnitude 
(except the $B_{2 g}$ frequency that changes from 930.5 to $941.0 \mathrm{~cm}^{-1}$ ). However, the individual changes are rather different for the harmonic and the actual fundamental frequencies meaning that transfering the basis set effect from the harmonic to the fundamental frequencies additively is not highly accurate.

At the cc-pVTZ level the introduction of diffuse functions in aug-cc-pVTZ has a rather large effect for two modes, but smaller effects are found at the cc-pVQZ level, where the cc-pVQZ and aug-cc-pVQZ results are similar within $4 \mathrm{~cm}^{-1}$.

Comparing our MP2/cc-pVXZ results with the CCSD(T)/cc-pVXZ results of Martin et all. $^{21,22}$, the MP2 results are seen to be around $40 \mathrm{~cm}^{-1}$ higher than the CCSD(T) results for the $\mathrm{CH}$-stretch modes. For the other modes the differences are less than $30 \mathrm{~cm}^{-1}$ and much smaller in most cases.

\section{E. Comparison between methods for large modal bases and comparison to exper-} iment

In Table VII we report calculations for the MP2/cc-pVQZ semi-quartic force field using VCI[gs,n] with $\mathrm{n}=1,2,3,4,5,6$ and $N_{m}=5$ VSCF modals per mode (up to 4737617 Hartree Products). Estimated from the previous benchmark calculations such a vibrational calculation is expected to be correct within about $2 \mathrm{~cm}^{-1}$ for the fundamental vibrations of ethylene for the given PES. This is certainly higher accuracy than the accuracy of the semi-quartic MP2/cc-pVQZ force field as clear from the previous sub-sections.

Comparing the $\mathrm{VCI}[\mathrm{gs}, 6]$ results for this PES to experiments we see an overestimation of the fundamental frequencies for the CH-stretch modes with up to $30 \mathrm{~cm}^{-1}$. For the other modes the discrepancies are less than $10 \mathrm{~cm}^{-1}$, which actually must be considered surprisingly good.

\section{SUMMARY AND OUTLOOK}

A number of different approaches for calculation of anharmonic vibrational energies for a given potential have been compared theoretically and for calculation of the fundamental vibrations of ethylene. FVCI calculations were used to benchmark approximate VCI approximations using both one common set of ground state VSCF one-mode functions for all states 
(VCI $[\mathrm{gs}, \mathrm{n}])$ and using a state specific approach (VCI[ss,n]) where the one-mode functions are optimized in VSCF calculations for each state. In addition VMP and VCC calculations were performed. Finally we have also shown that both four mode coupling terms and the fifthand sixth-order terms must be included in the truncated PES in order to obtain accurate results consistingly better than $20 \mathrm{~cm}^{-1}$ for all fundamental vibrations.

The convergence problems of VMP were demonstrated. However, the lowest order correction to VSCF, the VMP2 approach actually gave quite reasonable results. For VCI methods, on the other hand, convergence to FVCI is ensured increasing the VCI space, and this was also verified for both $\mathrm{VCI}[\mathrm{gs}, \mathrm{n}]$ and $\mathrm{VCI}[\mathrm{ss}, \mathrm{n}]$ as $\mathrm{n}$ approached the number of modes, even though the behavior for small $\mathrm{n}$ was not always very good. The performance of VCC was encouraging from the perspective that more accurate results are obtained compared to equivalent VCI calculation with the same number of parameters. The better performance of VCC is believed to be due to the exponential parameterization and the related size-extensivity of VCC, which will ultimately become increasingly important proceeding to systems with more degrees of freedom.

Though the behavior of VCC in many ways is encouraging, there were also problems that calls for further developments. Most importantly, the problems inherent to singlereference theories, which already were seen to cause some problems for the state specific VCI calculation, also caused serious problems in the VCC calculations. States were the VSCF reference state consituted less than $90 \%$ of the FVCI state had larger errors compared to the other states more dominated by the VSCF reference. This situation is rather similar to the situation in electronic structure theory were single-reference CC methods are known to give high accuracies when the Hartree-Fock description is a reasonable starting point, but can be expected to be less accurate for genuine multi-reference cases.

A related issues is that with the present implementation of VCC, it was a practical problems to solve the non-linear VCC equations for the problematic states mentioned above. This is probably related to that the iterative algorithm employed in the present implementation uses various partitionings in the solutions of the equations that one way or the other is related to perturbation theory which was found to diverge for these states. Obviously, it is a task for future research to improve the iterative algorithms for solving the equations for the VCC methods discussed here. Furthermore one may investigate more advanced ways of defining the excitation space giving new ways of defining truncated VCC methods. Some 
possibilities have already been opended for defining the cluster expansion in ways that are more flexible ${ }^{20}$ and potentially more balanced with respect to treating large contributions from more than one reference state. This may improve both the accuracy and make the procedure for solving the VCC equations more stable.

In electronic structure theory coupled cluster theory has proven to be an accurate method also for excited states. However, the commonly used CC approaches for excited electronic states are based not a state specific approach, but rather on response theoretical approaches $^{33,34}$ or related methods ${ }^{35}$ which circumvents the previously mentioned multireference problems to some extent. Response theory on the basis of VCC is promising with respect to both accuracy and simplicity since it maintains size-extensivity while avoiding non-linearity in the solution of equations for the excited states that caused problems in the state-specific VCC calculations. The development of vibrational response theory methods is currently underway, albeit the application of response theory in the context of VCC is not simple.

We shall conclude by stating that a number of methods for calculation of bound anharmonic vibrational states have been developed over the years, and a few of them have been discussed and tested in this paper. While more research are necessary on many issues, it is fair to say that the calculation of anharmonic vibrational wave functions has reached an advanced stage. There begins to be something to choose from with respect to adjusting the accuracy and the level of effort to the needs of a concrete application. We expect to see many more applications of such methods in the future and that they will approach more and more the status of being standard computational tools.

\section{ACKNOWLEDGMENTS}

This work has been supported by the Danish Research Council and DCSC (Dansk Center for Supercomputing). Josep Maria Luis acknowledges financial support from the Generalitat de Catalunya through the BE2004 program.

1 edited by P. Jensen and P. R. Bunker. Computational Molecular Spectroscopy. Wiley, Chichester, 2000. 
2 T.Seideman. Forming superposition states, in Computational Molecular Spectroscopy, edited by P. Jensen and P. R. Bunker, pp. 589-624. Wiley, Chichester, 2000.

3 D. M. Bishop, Adv. Chem. Phys. 104, 1 (1998).

4 J. M. Bowman, J. Chem. Phys. 68, 608 (1978).

5 J. M. Bowman, Acc. Chem. Res. 19, 202 (1986).

6 R. Gerber and M. Ratner, Adv. Chem. Phys. 70, 97 (1988).

7 R. Gerber and J. Jung. The vibrational self-consistent field approach and extensions: Method and applications to spectroscopy of large molecules and clusters, in Computational Molecular Spectroscopy, edited by P. Jensen and P. R. Bunker, pp. 365-390. Wiley, Chichester, 2000.

8 J. Bowman, K. M. Christoffel and F. Tobin, J. Phys. Chem. 83, 905 (1979).

9 K. M. Christoffel and J. M. Bowman, Chem. Phys. Letters. 85, 220 (1982).

10 S. Carter, J. M. Bowman and N. C. Handy, Theor. Chim. Acta. 100, 191 (1998).

11 C. Møller and M. S. Plesset, Phys. Rev. 46, 618 (1934).

12 L. S. Norris, M. A. Ratner, A. E. Roitberg and R. B. Gerber, J. Chem. Phys. 105, 11261 (1996).

13 J. O. Jung and R. B. Gerber, J. Chem. Phys. 105, 10332 (1996).

14 G. Chaban, J. O. Jung and R. B. Gerber, J. Chem. Phys. 111, 1823 (1999).

15 O. Christiansen, J. Chem. Phys. 119, 5773 (2003).

16 K. Yagi, K. Hirao, T. Taketsugu and M. Gordon, J. Chem. Phys. 121, 1283 (2004).

17 K. Yagi, T. Taketsugu, K. Hirao and M. S. Gordon, J. Chem. Phys. 113, 1005 (2000).

18 Thompson and D. Truhlar, Chem. Phys. Letters. 75, 87 (1980).

19 O. Christiansen, J. Chem. Phys. 120, 2140 (2004).

20 O. Christiansen, J. Chem. Phys. 120, 2149 (2004).

21 J. Martin, T. Lee, P. Taylor and J.-P. Francois, J. Chem. Phys. 103, 2589 (1995).

22 J. Martin and P. Taylor, Chem. Phys. Letters. 248, 336 (1996).

23 T. Lee, W. Allen and H. S. III, J. Chem. Phys. 87, 7062 (1987).

24 D. C. Jr., W. Allen, Y. Y. R.B. Remington and H. S. III, Chem. Phys. 123, 187 (1988).

25 N. Handy, C. Murray and R. Amos, J. Phys. Chem. 97, 4392 (1993).

26 W. Laidig, G. Fitzgerald and R. J. Bartlett, Chem. Phys. Lett. 113, 151 (1985).

27 T. Helgaker, P. Jørgensen and J. Olsen. Molecular electronic structure theory. Wiley, Chichester, 2000. 
28 P. Knowles, K. Somasundram, N. Handy and K. Hirao, Chem. Phys. Lett. 115, 8 (1985).

29 M. J. Frisch, G. W. Trucks, H. B. Schlegel, G. E. Scuseria, M. A. Robb, J. R. Cheeseman, J. A. Montgomery, Jr., T. Vreven, K. N. Kudin, J. C. Burant, J. M. Millam, S. S. Iyengar, J. Tomasi, V. Barone, B. Mennucci, M. Cossi, G. Scalmani, N. Rega, G. A. Petersson, H. Nakatsuji, M. Hada, M. Ehara, K. Toyota, R. Fukuda, J. Hasegawa, M. Ishida, T. Nakajima, Y. Honda, O. Kitao, H. Nakai, M. Klene, X. Li, J. E. Knox, H. P. Hratchian, J. B. Cross, V. Bakken, C. Adamo, J. Jaramillo, R. Gomperts, R. E. Stratmann, O. Yazyev, A. J. Austin, R. Cammi, C. Pomelli, J. W. Ochterski, P. Y. Ayala, K. Morokuma, G. A. Voth, P. Salvador, J. J. Dannenberg, V. G. Zakrzewski, S. Dapprich, A. D. Daniels, M. C. Strain, O. Farkas, D. K. Malick, A. D. Rabuck, K. Raghavachari, J. B. Foresman, J. V. Ortiz, Q. Cui, A. G. Baboul, S. Clifford, J. Cioslowski, B. B. Stefanov, G. Liu, A. Liashenko, P. Piskorz, I. Komaromi, R. L. Martin, D. J. Fox, T. Keith, M. A. Al-Laham, C. Y. Peng, A. Nanayakkara, M. Challacombe, P. M. W. Gill, B. Johnson, W. Chen, M. W. Wong, C. Gonzalez, and J. A. Pople. Gaussian 03, Revision C.02. Gaussian, Inc., Wallingford, CT, 2004.

30 T. Dunning, J.Chem.Phys. 90, 1007 (1989).

31 P. Davis and P. Rabinowitz. in Numerical Integration, edited by P. Jensen and P. R. Bunkerp. 166. Blaisdell, London, 1967.

32 O. Christiansen. MidasCpp (Molecular Interactions, dynamics and simulation in C++/Chemistry program package). University of Aarhus, 2004.

33 O. Christiansen, H. Koch, A. Halkier, P. Jørgensen, T. Helgaker and A. S. de Meras, J. Chem. Phys. 105, 6921 (1996).

34 H. J. Monkhorst, Int. J. Quantum Chem. S11, 421 (1977).

35 J. F. Stanton and R. J. Bartlett, J. Chem. Phys. 98, 7029 (1993). 


\section{FIGURE CAPTIONS:}

FIG. 1: Logarithm of VMP energies (in $\mathrm{cm}^{-1}$ ) in different orders of perturbation theory. 


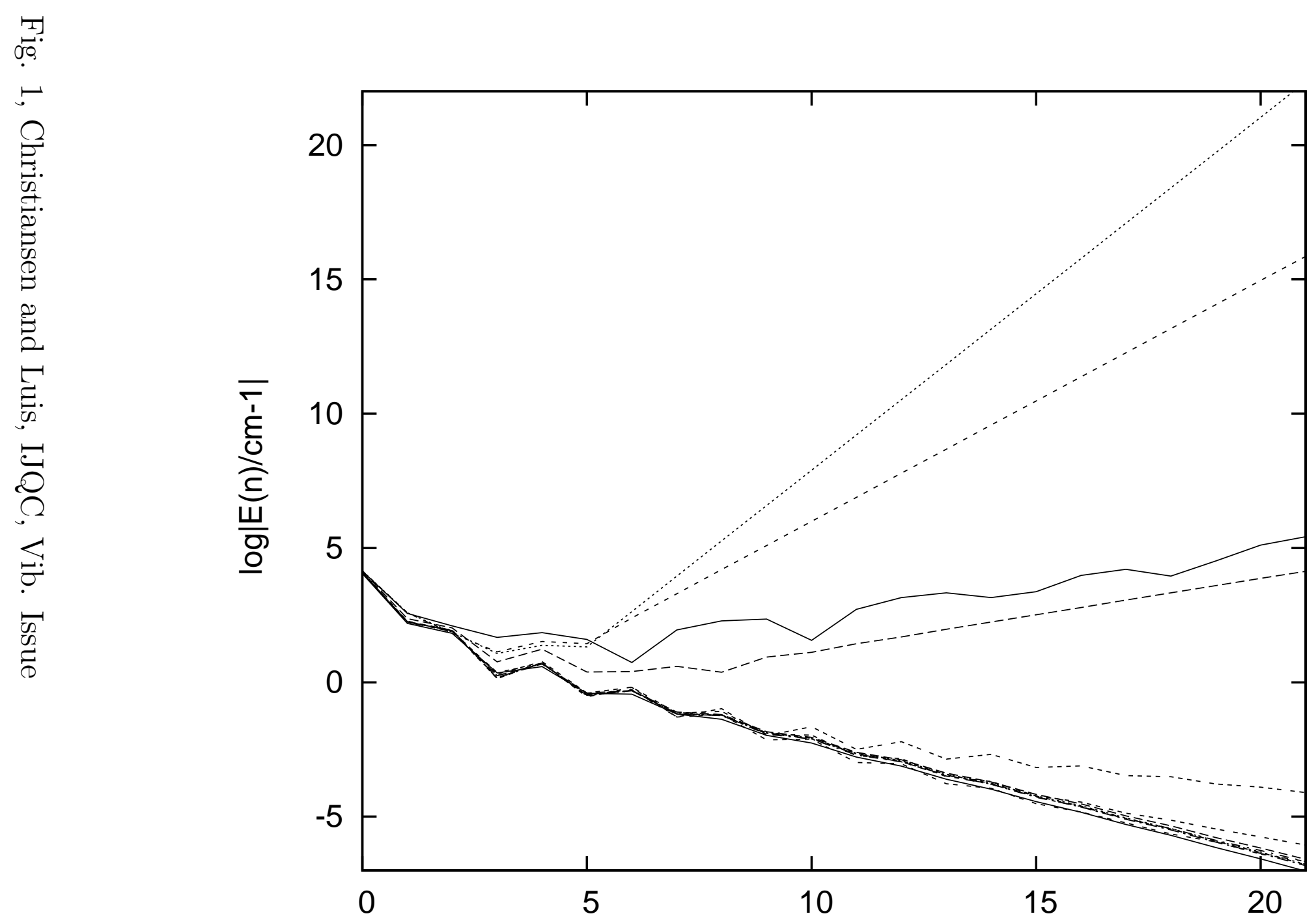


TABLE I: Computed fundamentals (in $\mathrm{cm}^{-1}$ ) for FVCI and errors relative to FVCI for both ground state VCI and state-specific VCI. The potential energy surface used is MP2/cc-pVTZ semi-quartic and $N_{m}=3 \mathrm{VSCF}$ modals were used per mode. MAD (MAXAD) is the mean(maximum) absolute deviation compared to FVCI[gs].

\begin{tabular}{|c|c|c|c|c|c|c|c|c|c|}
\hline & $\mathrm{FVCI}[\mathrm{gs}]$ & $\mathrm{VCI}[\mathrm{gs}, 1]$ & $\mathrm{VCI}[\mathrm{gs}, 2]$ & $\mathrm{VCI}[\mathrm{gs}, 3]$ & $\mathrm{VCI}[\mathrm{gs}, 4]$ & $\mathrm{VCI}[\mathrm{gs}, 5]$ & $\mathrm{VCI}[\mathrm{gs}, 6]$ & $\mathrm{VCI}[\mathrm{gs}, 7]$ & $\mathrm{VCI}[\mathrm{gs}, 8]$ \\
\hline & 3158.49 & 56.02 & 54.72 & 25.47 & 2.65 & 0.52 & 0.08 & 0.00 & 0.00 \\
\hline & 3141.80 & 46.61 & 49.93 & 22.26 & -1.83 & -8.63 & 0.20 & 0.01 & 0.00 \\
\hline & 3052.63 & 34.81 & 23.56 & 46.47 & 3.43 & 0.89 & 0.12 & 0.01 & 0.00 \\
\hline & 3019.53 & 88.47 & 53.69 & 37.11 & 11.67 & 0.86 & 0.21 & 0.01 & 0.00 \\
\hline & 1636.29 & 14.88 & 33.06 & 36.70 & 0.91 & 0.50 & 0.05 & 0.00 & 0.00 \\
\hline & 1439.05 & 21.90 & 36.95 & 35.40 & 1.20 & 0.49 & 0.04 & 0.00 & 0.00 \\
\hline & 1355.15 & 9.68 & 31.54 & 37.38 & 0.84 & 0.51 & 0.05 & 0.00 & 0.00 \\
\hline & 1221.94 & 17.66 & 35.61 & 34.47 & 0.90 & 0.45 & 0.04 & 0.00 & 0.00 \\
\hline & 1044.19 & 25.77 & 37.68 & 31.66 & 0.86 & 0.37 & 0.03 & 0.00 & 0.00 \\
\hline & 958.41 & 30.67 & 37.64 & 30.73 & 0.82 & 0.35 & 0.02 & 0.00 & 0.00 \\
\hline & 937.51 & 29.16 & 37.86 & 30.76 & 0.83 & 0.35 & 0.02 & 0.00 & 0.00 \\
\hline & 829.06 & 27.63 & 35.69 & 31.32 & 0.79 & 0.37 & 0.02 & 0.00 & 0.00 \\
\hline MAD & & 33.61 & 38.99 & 33.31 & 2.23 & 1.19 & 0.07 & 0.00 & 0.00 \\
\hline MAXAD & & 88.47 & 54.72 & 46.47 & 11.67 & 8.63 & 0.21 & 0.01 & 0.00 \\
\hline & FVCI[ss] & $\mathrm{VSCF}=\mathrm{VCI}[\mathrm{ss}, 1]$ & $\mathrm{VCI}[\mathrm{ss}, 2]$ & $\mathrm{VCI}[\mathrm{ss}, 3]$ & $\mathrm{VCI}[\mathrm{ss}, 4]$ & $\mathrm{VCI}[\mathrm{ss}, 5]$ & $\mathrm{VCI}[\mathrm{ss}, 6]$ & $\mathrm{VCI}[\mathrm{ss}, 7]$ & $\mathrm{VCI}[\mathrm{ss}, 8]$ \\
\hline & 3155.73 & 19.62 & 27.61 & 24.71 & 4.80 & 1.12 & 0.18 & 0.01 & 0.00 \\
\hline & 3130.73 & 17.66 & 25.87 & 44.55 & 9.60 & 3.24 & 0.47 & 0.05 & 0.00 \\
\hline & 3052.20 & 50.07 & 16.87 & 21.49 & 7.34 & 2.81 & 0.38 & 0.05 & 0.00 \\
\hline & 3018.14 & 54.71 & 62.25 & 39.52 & 14.34 & 4.07 & 0.32 & 0.04 & 0.00 \\
\hline & 1636.23 & 16.46 & 11.35 & 2.81 & 1.51 & 0.14 & 0.03 & 0.00 & 0.00 \\
\hline & 1438.95 & 20.19 & 19.79 & 2.00 & 1.14 & 0.34 & 0.02 & 0.00 & 0.00 \\
\hline & 1355.14 & 10.99 & 7.14 & 1.09 & 0.55 & 0.07 & 0.01 & 0.00 & 0.00 \\
\hline & 1221.82 & 15.81 & 15.48 & 0.93 & 0.59 & 0.12 & 0.01 & 0.00 & 0.00 \\
\hline & 1043.85 & 21.72 & 21.97 & 1.03 & 0.71 & 0.12 & 0.01 & 0.00 & 0.00 \\
\hline & 957.65 & 23.53 & 24.54 & 1.19 & 0.77 & 0.15 & 0.01 & 0.00 & 0.00 \\
\hline & 936.92 & 23.34 & 24.07 & 1.15 & 0.76 & 0.14 & 0.01 & 0.00 & 0.00 \\
\hline & 827.97 & 19.12 & 20.62 & 1.01 & 0.57 & 0.12 & 0.01 & 0.00 & 0.00 \\
\hline MAD & & 24.43 & 23.13 & 11.79 & 3.56 & 1.04 & 0.12 & 0.01 & 0.00 \\
\hline MAXAD & & 54.71 & 62.25 & 44.55 & 14.34 & 4.07 & 0.47 & 0.05 & 0.00 \\
\hline
\end{tabular}


TABLE II: Computed fundamentals $\left(\right.$ in $\mathrm{cm}^{-1}$ ) for FVCI[ss] and errors relative to FVCI[ss] for selected VMP and VCC methods. The potential energy surface used is MP2/cc-pVTZ semi-quartic and $N_{m}=3 \mathrm{VSCF}$ modals were used per mode. MAD (MAXAD) is the mean(maximum) absolute deviation compared to FVCI[ss].

\begin{tabular}{cccccccc}
\hline \hline & FVCI[ss] & VMP2[2] & VMP2[3] & VMP4[3] & VMP4[6] & VCC[ss,2] & VCC[ss,3] \\
\hline 3155.73 & 28.13 & 12.55 & 23.42 & 2.28 & 27.61 & 21.44 \\
& 3130.73 & 26.40 & 18.62 & 32.41 & 0.43 & 25.87 & 32.73 \\
3052.20 & 11.32 & 11.45 & 19.09 & 1.50 & 16.20 & $\left(16^{a}\right)$ \\
& 3018.14 & 62.73 & -4.53 & 115.96 & -26.96 & 62.25 & 33.71 \\
& 1636.23 & 11.31 & 2.73 & 2.50 & 0.49 & 10.88 & 1.50 \\
& 1438.95 & 19.79 & 2.60 & 1.99 & 0.33 & 19.79 & 1.44 \\
& 1355.14 & 7.12 & 1.48 & 1.05 & 0.26 & 7.01 & 0.63 \\
& 1221.82 & 15.53 & 1.70 & 0.93 & 0.18 & 15.48 & 0.68 \\
& 1043.85 & 22.10 & 2.23 & 1.03 & 0.20 & 21.97 & 0.74 \\
& 957.65 & 24.73 & 2.46 & 1.19 & 0.23 & 24.55 & 0.86 \\
& 936.92 & 24.25 & 2.41 & 1.16 & 0.22 & 24.08 & 0.82 \\
MAD & 827.97 & 20.75 & 1.52 & 0.98 & 0.13 & 20.63 & 0.78 \\
\hline \hline
\end{tabular}

${ }^{a}$ Not fully converged.

${ }^{b}$ Excluding the CH-stretch frequencies. 
TABLE III: Convergence of fundamental vibrations with respect to number of VSCF modals used per mode. Vibrational wave functions is $\operatorname{VCI}[\mathrm{gs}, 4]$ and the ground state VSCF modals are used ordered after increasing energy. Fundamental vibration energies obtained using 9 ground state VSCF modals per mode are given and the deviation with respect to these results using $3,4,5,6,7$ and 8 VSCF modals per mode. The potential energy surface used is the MP2/cc-pVTZ semiquartic. MAD (MAXAD) is the mean(maximum) absolute deviation compared to the $N_{m}=9$ results.

\begin{tabular}{lcccccc}
\hline \hline$[9]$ & {$[3]$} & {$[4]$} & {$[5]$} & {$[6]$} & {$[7]$} & {$[8]$} \\
\hline 3137.18 & 23.96 & 1.07 & 0.39 & 0.02 & 0.01 & 0.00 \\
3113.24 & 26.73 & 3.54 & 0.50 & 0.18 & 0.01 & 0.01 \\
3048.22 & 7.84 & -0.68 & 1.20 & 0.81 & 0.11 & 0.09 \\
3012.46 & 18.74 & 0.82 & 0.29 & 0.02 & 0.01 & 0.00 \\
1627.55 & 9.65 & 6.36 & 0.99 & 0.71 & 0.08 & 0.05 \\
1438.48 & 1.77 & 0.08 & 0.01 & 0.00 & 0.00 & 0.00 \\
1354.56 & 1.44 & 0.28 & 0.02 & 0.02 & 0.00 & 0.00 \\
1220.99 & 1.84 & 0.06 & 0.01 & 0.00 & 0.00 & 0.00 \\
1039.94 & 5.11 & 0.20 & 0.07 & 0.00 & 0.00 & 0.00 \\
949.67 & 9.56 & 0.48 & 0.25 & 0.02 & 0.01 & 0.00 \\
930.49 & 7.85 & 0.37 & 0.17 & 0.01 & 0.01 & 0.00 \\
817.64 & 12.21 & 0.70 & 0.46 & 0.04 & 0.03 & 0.00 \\
& & & & & & \\
MAD & 10.56 & 1.22 & 0.36 & 0.15 & 0.02 & 0.01 \\
MAXAD & 26.73 & 6.36 & 1.20 & 0.81 & 0.11 & 0.09 \\
\hline \hline
\end{tabular}


TABLE IV: Fundamental frequencies $\left(\mathrm{cm}^{-1}\right)$ calculated using various approximations for the PES. Electronic structure level was MP2/cc-pVDZ and the vibrational structure approximation was VCI[gs,4] with 9 modals per mode. Results are given as absolute numbers for the fundamental vibration for the semi-quartic force field and for the other PES as deviations from these numbers.

\begin{tabular}{|c|c|c|c|}
\hline semi-quartic & Romberg & full quartic & semi-quartic $+5+6$ \\
\hline 3145.2 & 0.2 & 18.6 & -22.7 \\
\hline 3127.4 & 0.2 & 16.2 & -23.7 \\
\hline 3048.7 & 0.2 & 23.0 & -7.7 \\
\hline 3011.1 & 0.1 & 11.7 & -18.6 \\
\hline 1647.1 & -0.1 & -0.3 & -0.8 \\
\hline 1421.4 & -0.0 & 1.7 & 0.0 \\
\hline 1344.9 & -0.0 & 2.4 & -0.4 \\
\hline 1207.0 & -0.0 & 1.8 & -0.7 \\
\hline 1027.4 & -0.2 & 0.3 & 9.7 \\
\hline 939.0 & -0.1 & -0.9 & 5.4 \\
\hline 903.6 & -0.1 & -0.5 & 5.4 \\
\hline 810.8 & -0.1 & -0.6 & -0.3 \\
\hline
\end{tabular}


TABLE V: Harmonic frequencies $\left(\mathrm{cm}^{-1}\right)$ for ethylene calculated at the MP2 level with different basis sets as compared to values from the literature.

\begin{tabular}{lcccccccc}
\hline \hline Sym. Number & $\begin{array}{c}\text { MP2/ } \\
\text { cc-pVDZ }\end{array}$ & $\begin{array}{c}\text { MP2/pVTZ } \\
\text { cc-pug-cc-pVTZ }\end{array}$ & cc-pVQZ & aug-cc-pVQZ & cc-pVTZ (Ref. 22) & cc-pVQZ (Ref. 22) \\
\hline$B_{2 u}$ & $\omega_{9}$ & 3311.2 & 3294.5 & 3288.6 & 3296.4 & 3293.8 & 3246.2 & 3248.0 \\
$B_{1 g}$ & $\omega_{5}$ & 3285.5 & 3267.7 & 3260.9 & 3270.5 & 3267.5 & 3219.3 & 3222.9 \\
$A_{g}$ & $\omega_{1}$ & 3206.2 & 3197.2 & 3191.9 & 3196.7 & 3193.4 & 3157.1 & 3156.9 \\
$B_{3 u}$ & $\omega_{11}$ & 3187.6 & 3179.3 & 3172.8 & 3180.7 & 3177.2 & 3139.1 & 3140.6 \\
$A_{g}$ & $\omega_{2}$ & 1687.4 & 1684.2 & 1677.8 & 1683.3 & 1680.4 & 1671.5 & 1671.6 \\
$B_{3 u}$ & $\omega_{12}$ & 1467.1 & 1484.8 & 1479.0 & 1481.9 & 1481.4 & 1479.0 & 1475.9 \\
$A_{g}$ & $\omega_{3}$ & 1375.8 & 1384.1 & 1379.6 & 1382.8 & 1381.3 & 1369.1 & 1368.3 \\
$B_{1 g}$ & $\omega_{6}$ & 1236.0 & 1246.7 & 1244.9 & 1247.4 & 1249.4 & 1242.0 & 1243.9 \\
$A_{u}$ & $\omega_{4}$ & 1065.4 & 1076.0 & 1047.1 & 1076.2 & 1072.9 & 1047.0 & 1049.2 \\
$B_{1 u}$ & $\omega_{7}$ & 972.8 & 983.0 & 975.5 & 981.2 & 979.9 & 966.8 & 965.4 \\
$B_{2 g}$ & $\omega_{8}$ & 935.8 & 959.6 & 945.7 & 964.1 & 963.9 & 941.8 & 948.0 \\
$B_{2 u}$ & $\omega_{10}$ & 825.5 & 827.8 & 823.3 & 827.7 & 827.9 & 822.9 & 823.5 \\
\hline \hline
\end{tabular}


TABLE VI: Fundamental frequencies $\left(\mathrm{cm}^{-1}\right)$ for ethylene calculated using semi-quartic PES obtained at the MP2 level with different basis sets. The vibrational calculations were VCI[gs,4] with $N_{m}=9$ ground state VSCF modals per mode.

\begin{tabular}{cccc}
\hline \hline MP2/cc-pVDZ & MP2/cc-pVTZ & MP2/aug-cc-pVTZ & MP2/cc-pVQZ \\
\hline 3145.4 & 3137.2 & 3134.4 & 3138.2 \\
3127.6 & 3113.2 & 3115.7 & 3118.0 \\
3048.9 & 3048.2 & 3045.3 & 3047.7 \\
3011.3 & 3012.5 & 3009.5 & 3012.7 \\
1647.1 & 1627.6 & 1623.6 & 1628.2 \\
1421.4 & 1438.5 & 1437.3 & 1438.0 \\
1344.9 & 1354.6 & 1350.7 & 1354.2 \\
1207.0 & 1221.0 & 1225.6 & 1224.3 \\
1027.3 & 1039.9 & 1116.4 & 1041.0 \\
938.9 & 949.7 & 951.3 & 949.8 \\
903.5 & 930.5 & 982.9 & 941.0 \\
810.7 & 817.6 & 818.1 & 818.6 \\
\hline \hline
\end{tabular}


TABLE VII: Fundamental frequencies $\left(\right.$ in $\mathrm{cm}^{-1}$ ) calculated VCI[gs,6], deviation from these results for $\operatorname{VCI}[\mathrm{gs}, \mathrm{n}]$ with $\mathrm{n}=1-5$, and experimental fundamental frequencies as compiled in Ref.21. A semi-quartic force-field calculated at the MP2/cc-pVQZ level were used and up to $N_{m}=5$ ground state VSCF modals per mode were used in the VCI calculations. MAD (MAXAD) is the mean(maximum) absolute deviation compared to $\mathrm{VCI}[\mathrm{gs}, 6]$.

\begin{tabular}{ccccccc}
\hline \hline & VCI[gs,6] & VCI[gs,1] & VCI[gs,2] & VCI[gs,4] & VCI[gs,5] & Exp. \\
\hline 3134.59 & 81.17 & 61.03 & 4.01 & 0.76 & 3104.89 \\
& 3109.38 & 80.51 & 65.86 & 9.07 & 2.14 & 3083.36 \\
3042.33 & 43.97 & 28.24 & 6.89 & 1.11 & 3022.03 \\
& 3002.39 & 105.68 & 58.37 & 10.62 & 0.94 & 2988.64 \\
& 1627.09 & 23.88 & 39.74 & 2.02 & 0.57 & 1625.4 \\
& 1436.22 & 24.16 & 38.00 & 1.77 & 0.52 & 1442.47 \\
& 1352.91 & 11.71 & 32.43 & 1.33 & 0.51 & 1343.54 \\
& 1222.89 & 20.01 & 36.43 & 1.41 & 0.47 & 1222 \\
& 1039.67 & 31.14 & 38.89 & 1.42 & 0.39 & 1025.59 \\
& 948.59 & 40.58 & 39.41 & 1.45 & 0.39 & 948.77 \\
& 939.72 & 36.85 & 39.28 & 1.43 & 0.39 & 939.86 \\
MAD & 817.62 & 40.43 & 37.70 & 1.46 & 0.40 & 825.93 \\
\hline \hline
\end{tabular}

